\title{
Study of a WIMP dark matter model with the updated results of CDMS II
}

\author{
Lian-Bao Jia and Xue-Qian Li* \\ Department of Physics, Nankai University, \\ Tianjin 300071, China
}

\begin{abstract}
The new observation of CDMS II favors low mass WIMPs. Taking the CDMS II new results as inputs, we consider a SM singlet: the darkon as the dark matter candidate, which can be either scalar, fermion or vector. It is found that the simplest scenario of $\mathrm{DM}+\mathrm{SM}$ conflicts with the stringent constraint set by the LHC data. New physics beyond the SM is needed, and in this work, we discuss an extended standard model $S U_{L}(2) \otimes U_{Y}(1) \otimes U(1)^{\prime}$ where $U(1)^{\prime}$ only couples to the darkon. The new gauge symmetry is broken into $U_{e m}(1)$ and two neutral bosons $Z^{0}$ and $Z^{\prime}$ which are mixtures of $W_{\mu}^{3}, B_{\mu} X_{\mu}$ are resulted in. Following the literature and based on the CDMS data, we make a complete analysis to testify the validity of the model. The cross section of the elastic scattering between darkon and nucleon is calculated, and the DM relic density is evaluated in the extended scenario as well. It is found that considering the constraints from both cosmology and collider experiments, only if $Z^{\prime}$ is lighter than $Z^{0}$, one can reconcile all the presently available data.
\end{abstract}

*Electronic address: lixq@nankai.edu.cn 


\section{INTRODUCTION}

Recently, the CDMS Collaboration reports that three WIMP-candidate events were observed [1] by using the silicon detectors. With a final surface-event background estimate of $0.41_{-0.08}^{+0.20}$ (stat. $)_{-0.24}^{+0.28}$ (syst.), they indicate that the highest likelihood occurs for a WIMP mass of 8.6 GeV/c $/ c^{2}$ and spin-independent WIMP-nucleon cross section of $1.9 \times 10^{-41} \mathrm{~cm}^{2}$. This observation seems to contradict with the results of XENON100 [2]. Hooper [3] re-analyzed the data of XENON100 and reached a different conclusion, namely the two experimental results can be reconciled. Therefore in this work, we take the CDMS results as inputs to study the dark matter. We will test a viable model proposed in literature, namely check whether both the astronomical observation and constraints from the collider experiments can be simultaneously satisfied in this scenario.

As is well known, none of the standard model (SM) particles can meet the criterion to stand as dark matter (DM) candidates. Many particles beyond the SM are proposed, for example the primordial black holes, axions, heavy neutrinos, the lightest supersymmetric neutralino, etc. Among them, the darkon model namely a SM singlet scalar [4 9] which interacts with the SM particles by exchanging Higgs boson only, probably is the simplest version for the dark matter candidates. The spin-independent cross section for the darkon-nucleon elastic scattering might be measured by the earth detectors. The typical recoil energy is $\Delta E_{R} \sim(\mu v)^{2} / m_{A}$, where $\mu$ is dark matter-nucleus reduced mass, $v$ is DM velocity, and $m_{A}$ is target nucleus mass. The WIMPs with not very heavy masses will weaken the bounds in detector search, and the low mass WIMPs (mass around $10 \mathrm{GeV}$ ) are more of our concern in this work.

Thanks to the successful operation of LHC where the Higgs boson signals have been observed [10, 11], it provides a possible means to directly detect the dark matter particles on the earth if they indeed exist. It means that all the proposed dark matter candidates and possible new interactions by which the DM particles interfere with our detector would withstand the stringent test on the earth colliders. Namely, if the proposed DM particles, especially the lighter ones, are not observed at LHC as expected, the concerned model fails or needs to be modified. As indicates in [7], if the mass of the darkon is lighter than half of Higgs mass, Higgs would decay into a darkon pair which is a channel with invisible final products, and the simplest version of scalar darkon+SM may fail. That is to say, if darkon's coupling to Higgs is not much smaller than 1, a large partial width is expected and it obviously contradicts to the measured value of the invisible width of the SM Higgs. As a possible extension of the scalar darkon+SM version, the two-Higgs-doublet model was discussed in [7, 8, 12] and there seems to be a large parameter space to accommodate both the LHC data on Higgs and the CDMS observations.

We also find that the scenario of darkon+SM, no matter the darkon is scalar, fermionic or vector, definitely fails, thus a new interaction beyond the SM is needed. Alternatively, we propose an interaction beyond the SM as the darkon+SM+an extra $U(1)^{\prime}$. The extended gauge group $S U_{L}(2) \otimes U_{Y}(1) \otimes U(1)^{\prime}$ breaks into $U_{e m}(1)$ and two neutral bosons $Z^{0}$ and $Z^{\prime}$ are resulted in. $Z^{0}$ and $Z^{\prime}$ are mixtures of $W_{\mu}^{3}, B_{\mu}$ and $X_{\mu}$ which is the gauge boson of the newly introduced $U(1)^{\prime}$, while the photon remains massless. In this scenario, to be consistent with the CDMS and LHC data simultaneously, we should assume that the coupling between the darkon and Higgs 


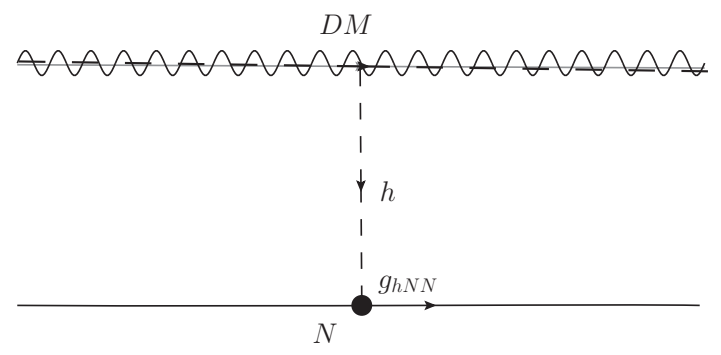

FIG. 1: The elastic scattering between dark matter and nucleon with Higgs boson exchanged.

boson to be very small and the interaction by exchanging Higgs between the detector material and darkon can be safely ignored. Therefore, the possible decays of Higgs boson into darkons are almost forbidden and one cannot expect to measure the mode at LHC at all. The scattering between the darkon and nucleons is due to exchanging the gauge boson $Z^{0}$ and $Z^{\prime}$. Definitely, such interaction may also exist in the decays of quarkonia, i.e. if the measurement of heavy quarkonia, such as bottomonia, are very precise, one may observe their decays into invisible final products besides the SM neutrino-anti-neutrino pairs. But it is estimated that the branching ratios for such decays of heavy quarkonia are too small to be reliably measured in any of our present facilities. Besides, when the bottomonia are lighter than the new invisible final products, these decays are also forbidden. Therefore this proposed darkon $+\mathrm{SM}+U(1)^{\prime}$ is safe with respect to the present experimental constraints. Moreover, the observed relic density of dark matter in our universe sets one more constraint on our model parameter space.

This work is organized as follows. After this introduction, we first consider the simple version of scalar, fermionic, and vector darkon within the framework of standard model plus darkon, then we derive the formulas of the cross section between nucleon and darkon, as well as the decay width of Higgs into invisible darkons. We further derive the corresponding formulas for the aforementioned extended version darkon $+\mathrm{SM}+U(1)^{\prime}$. Then in the following section, we numerically evaluate the cross sections of darkon-nucleon elastic scattering with the two scenarios. We indicate that the simple version does not satisfy the constraint set by the LHC data as long as we take the CDMS data as inputs, but in the extended version there is a parameter space to accommodate both the experimental measurements. The last section is devoted to our brief summary and discussions.

\section{DARKON+SM}

In this work, as CDMS data suggested, we focus on low mass WIMPs. The WIMP particle could be an $S U_{c}(3) \times S U_{L}(2) \times U_{Y}(1)$ singlet, i.e. either a scalar or fermion or vector darkon [7, 13, 14]. In the scenario of darkon+SM, the elastic scatting between darkon and the detector material is realized via t-channel Higgs exchange, as described in Fig. 1, 


\section{A. Scalar darkon}

Let us consider a scalar type WIMP DM, namely a scalar darkon first. This type DM has been discussed in [7], and here for completeness we first repeat some relevant procedures. The Lagrangian is written as [4-7]

$$
\mathcal{L}=\mathcal{L}_{\mathcal{S M}}-\frac{\lambda_{S}}{4} S^{4}+\frac{1}{2} \partial^{\mu} S \partial_{\mu} S-\frac{m_{0}^{2}}{2} S^{2}-\lambda S^{2} H^{\dagger} H
$$

Here $\lambda_{S}, m_{0}$, and $\lambda$ are free parameters to be determined by fitting data. It has been indicated in earlier works that the scalar darkon field has no mixing with the Higgs field, and this can avoid fast decaying into SM particles because dark matter particles must be sufficiently stable and survive from the Big Bang to today. From Eq. (1), the SM singlet scalar darkon can be further written as

$$
\mathcal{L}_{\mathcal{S}}=-\frac{\lambda_{S}}{4} S^{4}+\frac{1}{2} \partial^{\mu} S \partial_{\mu} S-\frac{m_{0}^{2}+\lambda v^{2}}{2} S^{2}-\frac{1}{2} \lambda S^{2} h^{2}-\lambda v S^{2} h .
$$

The Higgs-nucleon coupling $g_{h N N}$ is needed in calculating the scatting process, $\mathcal{L}_{h N N}=$ $-g_{h N N} \bar{N} N h$. Here we adopt the value of $g_{h N N}$ given by He et al. [8],

$$
g_{h N N} \bar{N} N=<N\left|\frac{k_{u}}{v}\left(m_{u} \bar{u} u+m_{c} \bar{c} c+m_{t} \bar{t} t\right)+\frac{k_{d}}{v}\left(m_{d} \bar{d} d+m_{s} \bar{s} s+m_{b} \bar{b} b\right)\right| N>
$$

and $g_{h N N} \simeq 1.71 \times 10^{-3}$. The cross-section of scalar DM-nucleon elastic scatting is [4-6]

$$
\sigma_{\mathrm{el}} \simeq \frac{\lambda^{2} v^{2} g_{h N N}^{2} m_{N}^{2}}{\pi\left(p_{D}+p_{N}\right)^{2} m_{h}^{4}}
$$

Here, $p_{D}, p_{N}$ are the momenta of the initial DM and nucleon. For low energy elastic scatting, $\left(p_{D}+p_{N}\right)^{2} \simeq\left(m_{D}+m_{N}\right)^{2}$, and $m_{D}, m_{N}$ are masses of DM, nucleon respectively. Substituting the darkon mass $8.6 \mathrm{GeV}$ and the cross section $1.9 \times 10^{-41} \mathrm{~cm}^{2}$ as given by CDMS II into the above formula (4), we can fix the effective coupling of Higgs-darkon.

The Higgs signals have been observed at LHC [10, 11] and $m_{h}=125 \mathrm{GeV}$, so by the data of CDMS, $\lambda \approx 0.148$ is determined. The partial width of Higgs decaying into two scalar darkons is

$$
\Gamma_{h \rightarrow S S}=\frac{\lambda^{2} v^{2}}{8 \pi m_{h}} \sqrt{1-\frac{4 m_{D}^{2}}{m_{h}^{2}}} .
$$

Substituting Higgs mass into the equation, $\Gamma_{h \rightarrow S S} \approx 0.418 \mathrm{GeV}$ is obtained. The main decay channel in SM is $h \rightarrow b \bar{b}$. In the Born approximation, the width of this channel is [15, 16]

$$
\Gamma_{\text {Born }}(h \rightarrow b \bar{b})=\frac{3 G_{F}}{4 \sqrt{2} \pi} M_{h} m_{b}^{2} \beta_{b}^{3} .
$$

Here $\beta=\sqrt{1-4 m_{b}^{2} / M_{h}^{2}}$, and $G_{F}$ is the Fermi coupling constant. With $G_{F}=1.166 \times 10^{-5} \mathrm{GeV}^{-2}$ and $m_{b}(\overline{M S})=4.18 \mathrm{GeV}$, we can obtain $\Gamma_{\text {Born }}(h \rightarrow b \bar{b}) \approx 0.00427 \mathrm{GeV}$. Thus the branching ratio $B_{h} \rightarrow$ invisible would be too large. 


\section{B. Fermionic and vectorial darkons}

In the spin- $\frac{1}{2}$ darkon+SM scenario, the effective interaction can be written as

$$
\mathcal{L}_{i n t}=-\lambda \bar{\psi}_{D} \psi_{D} h
$$

The cross-section of the low energy fermion-darkon-nucleon elastic scatting is

$$
\sigma_{\mathrm{el}} \simeq \frac{\lambda^{2} m_{D}^{2} g_{h N N}^{2} m_{N}^{2}}{\pi\left(p_{D}+p_{N}\right)^{2} m_{h}^{4}} .
$$

The partial width of Higgs decaying into two darkon spinors is

$$
\Gamma_{h \rightarrow D M}=\frac{\lambda^{2} m_{h}}{8 \pi} \sqrt{1-\frac{4 m_{D}^{2}}{m_{h}^{2}}} .
$$

In this case, the invisible decay width is unbearably large when $\lambda$ is at order of unity. It means that such spin- $\frac{1}{2}$ darkon+SM scenario must also be abandoned.

For a vector darkon, the effective lagrangian can be written as

$$
\mathcal{L}_{V H}=\lambda V^{\mu} V_{\mu} H^{\dagger} H
$$

The cross section of the elastic scattering between a vector darkon and nucleon via a Higgs boson exchange is

$$
\sigma_{\mathrm{el}} \simeq \frac{\lambda^{2} v^{2} g_{h N N}^{2} m_{N}^{2}}{\pi\left(p_{D}+p_{N}\right)^{2} m_{h}^{4}}
$$

The numerical results for the vector darkon are similar to the two above cases for scalar and fermion darkons, namely with the darkons possessing a low mass of order of $10 \mathrm{GeV}$ and the spin-independent cross section as determined by the CDMS data, the partial width of Higgs decaying into invisible final products would be too large to be tolerated.

The above results indicate that the simplest scenario of darkon+SM, no matter the SM singlet darkon is a scalar, fermion or vector, cannot reconcile the cosmological observation of CDMS and the LHC data. Then one should invoke an extended version of SM i.e. a darkon+SM+BSM scenario. But what model beyond standard model (BSM) which can be applied to explain the CDMS observation and the LHC data simultaneously, is a problem. There are many different proposals, and below we will investigate a naturally extended version of the SM, i.e. introducing an extra $U(1)^{\prime}$ gauge field which would be broken and a new vector boson $Z^{\prime}$ is induced.

\section{DARKON+SM+U(1)}

For low mass darkon model, the simple version darkon+SM where darkons interact with the SM particles in detector by exchanging Higgs boson at t-channel, definitely fails to reconcile 
the observation of CDMS and LHC data, and therefore needs to be modified. To tolerate the CDMS and LHC data, besides the two-Higgs-doublet model mentioned above, alternatively, for example, the sneutrino dark matter which interacts with the detector material dominantly via exchanging SM Z-boson, was discussed in [17].

In this work, we would study the effects of an extended SM by adding an extra $U(1)^{\prime}$ [18 24] which only interacts with the darkons (no matter scalar, fermion or vector darkons) into the gauge group, as $S U_{L}(2) \otimes U_{Y}(1) \otimes U(1)^{\prime}$ whose gauge bosons are respectively $W_{\mu}^{ \pm}, W_{\mu}^{3}, B_{\mu}$ and $X_{\mu}$ (for more discussions about this model, see e.g., [25 29]). The extended symmetry later breaks into $U_{\text {em }}(1)$. As a consequence, besides the regular charged $W^{ \pm}$, two neutral gauge bosons $Z^{0}$ and $Z^{\prime}$ gain masses after the symmetry breaking while the photon remains massless.

It is noted that a small mixing between the SM $Z$ and $X$ results in the physical mass eigenstates $Z^{0}$ and $Z^{\prime}$. Since the mixing is required to be very small the resultant $Z^{0}=\cos \varphi Z+\sin \varphi X$ is almost the SM $Z$ boson whereas $Z^{\prime}$ is overwhelmingly dominated by $X$. Concretely, after $S U(2)_{L} \times U(1)_{Y} \times U(1)^{\prime}$ breaking, one has

$$
\left(\begin{array}{c}
A_{\mu} \\
Z_{\mu}^{0} \\
Z_{\mu}^{\prime}
\end{array}\right)=\left[\begin{array}{ccc}
\cos \theta_{w} & \sin \theta_{w} & 0 \\
-\sin \theta_{w} \cos \varphi & \cos \varphi \cos \theta_{w} & \sin \varphi \\
\sin \theta_{w} \sin \varphi & -\sin \varphi \cos \theta_{w} & \cos \varphi
\end{array}\right]\left(\begin{array}{c}
B_{\mu} \\
W_{\mu}^{3} \\
X_{\mu}
\end{array}\right) .
$$

Assuming $X_{\mu}$ of $U(1)^{\prime}$ only couples to the darkon but not the SM particle whereas $Z_{\mu}$ only couples to the SM particles, thus the interaction between the darkon and SM particles must be realized via the small mixing. Namely the effective interaction amplitude between the darkon and protons or neutrons in the earth detector must be proportional to $\sin \varphi \cdot \cos \varphi$. To be consistent with experiments, $\varphi$ should be very small, i.e. $\sin \varphi \ll 1, \cos \varphi \sim 1$.

Since the new effective vertex $V_{D V(A) D}$ is a coupling between scalar, fermionic or vector darkon with the gauge boson, the Lorentz structure are well determined even though the coupling constants might be model dependent. Feytsis and Ligeti [30] listed all possible operators and indicated which one(s) is suppressed by $q^{2}$ or $v^{2}$ where $q$ is the exchanged momentum and $v$ is the speed of the dark matter relative to the earth detector. Thus, in this work, we only concern the unsuppressed spin-independent scattering processes which may correspond to the recently observed events. Below, we will be focusing on the fermionic darkon and give all the details, but for completeness we also briefly discuss the cases for the scalar and vector darkons.

\section{A. Fermionic darkon}

Let us consider the fermionic darkon first. The axial-vector component of the gauge boson may induce a fermionic darkon-nucleon interaction which is not suppressed by $q^{2}$ or $v^{2}$, even though this coupling would result in a spin-dependent cross section [30]. For easily handling, here we consider a right-handed darkon with the vertex $i \lambda \gamma^{\mu} \frac{1+\gamma^{5}}{2}$ to interact with the SM particles via exchanging Z-boson. The darkon-nucleon elastic scattering cross section is calculated for two cases: $m_{Z^{\prime}} \gg m_{Z^{0}}$ and $m_{Z^{\prime}} \ll m_{Z^{0}}$ respectively, and corresponding DM relic density is also computed. 


\section{The case $m_{Z^{\prime}} \gg m_{Z^{0}}$}

In this case, the darkon-nucleon elastic scattering occurs mainly via exchanging $Z^{0}$. The fugacity speed of WIMP is about $220 \sim 544 \mathrm{~km} / \mathrm{s}$ [31]. For the low energy $Z^{0}$-nucleon interaction, the hadronic matrix element can be expressed as [32 34$]$

$$
\left\langle p^{\prime}, s^{\prime}\left|J_{\mu}^{Z^{0}}\right| p, s\right\rangle=\sqrt{\frac{G_{F}}{\sqrt{2}}} \overline{\mathcal{U}}_{N}\left(p^{\prime}, s^{\prime}\right)\left[G_{A}^{z} \gamma_{\mu} \gamma^{5}+F_{1}^{z} \gamma_{\mu}+F_{2}^{z} \frac{i \sigma_{\mu \nu} q^{\nu}}{2 M_{N}}\right] \mathcal{U}_{N}(p, s) .
$$

Here $\mathcal{U}_{N}, M_{N}, q$ are the nucleon's wave function, mass, and momentum transfer respectively. $G_{A}^{z}, F_{1}^{z}$, and $F_{2}^{z}$ are the relevant form factors. Those form factors can be determined by the data of elastic scattering between neutrino and nucleon, since for this neutral current scattering process only $Z^{0}$ exchange is dominant (the new boson $Z^{\prime}$ is suppressed by a factor $\sin ^{4} \varphi$ in this process).

Here we adopt the way given in Ref. [32, 33] to define the form factors. In the form of quark currents, the hadronic matrix element is written as

$$
\left\langle p^{\prime}, s^{\prime}\left|J_{\mu}^{Z^{0}}\right| p, s\right\rangle=\sqrt{\frac{G_{F}}{\sqrt{2}}} \overline{\mathcal{U}}_{N}\left(p^{\prime}, s^{\prime}\right) \sum_{i}\left[\bar{q}_{i} \gamma_{\mu}\left(1-\gamma^{5}\right) t_{z} q_{i}-2 Q_{i} \sin ^{2} \theta_{w} \bar{q}_{i} \gamma_{\mu} q_{i}\right] \mathcal{U}_{N}(p, s) .
$$

The form factors are written as

$$
\begin{gathered}
G_{A}^{z}=-\frac{G_{A}^{3} \tau_{3}}{2}+\frac{G_{A}^{s}}{2}, \\
F_{1}^{z}=\left(1-2 \sin ^{2} \theta_{w}\right) F_{1}^{3} \tau_{3}-2 \sin ^{2} \theta_{w} F_{1}^{1}-\frac{F_{1}^{s}}{2}, \\
F_{2}^{z}=\left(1-2 \sin ^{2} \theta_{w}\right) F_{2}^{3} \tau_{3}-2 \sin ^{2} \theta_{w} F_{2}^{1}-\frac{F_{2}^{s}}{2},
\end{gathered}
$$

where the isospin factor $\tau_{3}=+(-)$ for proton (neutron), and

$$
\begin{gathered}
F_{j}^{1}=\frac{F_{j}^{p}+F_{j}^{n}}{2}, \\
F_{j}^{3}=\frac{F_{j}^{p}-F_{j}^{n}}{2},
\end{gathered}
$$

with $\mathrm{j}=1,2$.

Defining $Q^{2}=-q^{2}$, since $Q^{2} / m_{N}^{2} \ll 1$, for darkon-nucleon scattering via exchanging $Z^{0}$ boson, we can set the values of the form factors at $Q^{2}=0$. At $Q^{2}=0, F_{1}^{p}=1, F_{1}^{n}=$ $0, F_{2}^{p}=1.7928, F_{2}^{n}=-1.9130$ [33]. In the limit of $Q^{2}=0$, the parameters corresponding to the strange part are $G_{1}^{s}(0)=\Delta s, F_{1}^{s}(0)=0, F_{2}^{s}(0)=\mu_{s}$ [33 35], and here we take the fitted results $G_{1}^{s}(0)=-0.15 \pm 0.07, F_{1}^{s}(0)=0, F_{2}^{s}(0)=0, M_{A}=1.049 \pm 0.019,\left(\chi^{2}=9.73\right.$ at 13 DOF) [33, 34]. The PDG average value of $G_{A}^{3}$ is $G_{A}^{3}=1.2701 \pm 0.0025$ [36]. So at $Q^{2}=0$, the form factors are $G_{A}^{z} \approx-0.710$ (0.560) for proton (neutron), and $F_{1}^{z}=0.5-2 \sin ^{2} \theta_{w}(-0.5)$ for proton (neutron). The contribution from $F_{2}^{z}$ term is suppressed at $Q^{2}=0$. If considering the conservation of the vector currents and just using the valence quarks in the nucleon, the same result can be obtained for the vector form factor $F_{1}^{z}$. 
As the darkon is non-relativistic, in the limit $\frac{P^{\mu}}{m} \rightarrow(1, \epsilon)$, the darkon-nucleon elastic scattering cross section with $Z^{0}$ exchanged at t-channel can be written as

$$
\sigma_{\mathrm{el}} \simeq \frac{\sqrt{2} G_{F} \lambda^{2} \sin ^{2} \varphi m_{D}^{2} m_{N}^{2}\left(3 G_{A}^{z^{2}}+F_{1}^{z^{2}}\right)}{4 \pi\left(p_{D}+p_{N}\right)^{2} m_{Z^{0}}^{2}} .
$$

It is noted that $G_{A}^{z}$ is spin-dependent (SD), and $F_{1}^{z}$ is spin-independent (SI). For large mass target nuclei, such as the silicon, germanium and xenon targets, the spin-independent interaction is enhanced by the atomic number $A^{2}$ (but not exactly, see below for details) in the target nucleus, so the spin-independent interaction is more sensitive than the spin-dependent case, as discussed in Ref. [30]. Thus we can drop the spin-dependent term $G_{A}^{z}$ but just keep the spin-independent term $F_{1}^{z}$ for large mass target nuclei scattering. For proton, $F_{1}^{z}(p)=0.5-2 \sin ^{2} \theta_{w} \approx 0.038$, while for neutron $F_{1}^{z}(n)=-0.5$. Thus, the darkon-neutron scattering is dominant and the scattering cross section of darkon-nucleus via exchanging a neutral gauge boson $Z$ should be proportional to $(A-Z)^{2}$ instead of $A^{2}$. Thus a factor of about 0.25 might exist and when analyzing the data to extract the information about the dark matter-nucleon interaction, this factor should be considered.

Substituting the CDMS II results for darkon-neutron elastic scattering: $m_{D} \sim 8.6 \mathrm{GeV} / c^{2}$ and the elastic cross section $\sigma_{\mathrm{el}} \sim 4 \times 1.9 \times 10^{-41} \mathrm{~cm}^{2}$ into the relevant formulas, we obtain $\lambda^{2} \sin ^{2} \varphi \approx 6.88 \times 10^{-3}$. To require the coupling constant $\alpha_{D}=\frac{\lambda^{2}}{4 \pi}<1$, the upper limit of $\lambda$ is $\sqrt{4 \pi}$.

In fact, the LEP data set a stringent constraint on the coupling and mixing. The width of $Z^{0}$ decaying into invisible products is $\Gamma$ (invisible $)=499.0 \pm 1.5 \mathrm{MeV}[36$ ]. It is assumed in our scenario, that subtracting the main contribution of neutrinos from the measured width, the rest can be attributed to the darkon products. Thus we can use the data to estimate the range of $\varphi$ with some unavoidable uncertainties. The width of $Z^{0}$ decaying into a darkon pair is formulated as

$$
\sin ^{2} \varphi \Gamma_{D}=\frac{\lambda^{2} \sin ^{2} \varphi\left(m_{Z^{0}}^{2}-m_{D}^{2}\right)}{24 \pi m_{Z^{0}}} \sqrt{1-\frac{4 m_{D}^{2}}{m_{Z^{0}}^{2}}}
$$

Then the total width of $Z^{0}$ decaying into invisible products is

$$
\cos ^{2} \varphi \Gamma_{\nu \bar{\nu}}+\sin ^{2} \varphi \Gamma_{D} \leq \Gamma_{\nu \bar{\nu}}+\sin ^{2} \varphi \Gamma_{D} \approx 505.7 \mathrm{MeV}
$$

and this value is larger than the experimentally measured value (the central value) for invisible products. If the mixing angle $\sin ^{2} \varphi$ is reduced to an order of 0.01 , this could satisfy LEP data. However, this mixing angle is too large to be accepted because the SM electroweak sector would be seriously affected to conflict with all the previous well-done measurements.

Another constraint comes from the observed density of dark matter in our space.

The motion of the darkon is non-relativistic, the invariant mass of a darkon pair can be approximated as $\sqrt{s} \simeq 2 m_{D}$ where $m_{D}$ is the darkon mass. In order to get the DM relic density, we need to calculate DM annihilation cross section. In Ref [6], the scalar-mediated (Higgs) $2 \rightarrow 2$ annihilation cross-section of DM pair into SM particles is given. But as discussed in the 
introduction, we choose an alternative scenario where the coupling of Higgs boson with darkon is too small to make any substantial contributions to the darkon-nucleon scattering and as well as the dark matter annihilation.

Here, the annihilation cross section of darkons is dominated by the process that a darkon pair annihilates into a virtual gauge boson $\left(Z^{0}\right.$ or $\left.Z^{\prime}\right)$ which later transits into SM final states. Considering the case that the intermediate boson has a narrow width compared with its mass at the pole, the cross section is written as

$$
\begin{aligned}
\sigma_{\mathrm{ann}}= & \frac{1}{2} \sigma_{\mathrm{ann}}^{\text {Dirac }}=\frac{1}{2} \frac{1}{\beta_{i}\left(2 s_{1}+1\right)\left(2 s_{2}+1\right)} \frac{\lambda^{2} \sin ^{2} \varphi \cos ^{2} \varphi}{\left(s-M^{2}\right)^{2}+M^{2} \Gamma^{2}} \\
& \times\left[2\left(s-m_{D}^{2}\right) \frac{\tilde{\Gamma}_{f}}{\sqrt{s}}+\left(\frac{s}{M^{2}}-1\right)^{2} \frac{m_{Z^{0}}^{2} G_{F}}{2 \sqrt{2}} \frac{N_{c} \beta_{f} c_{a}^{2} m_{f}^{2} m_{D}^{2}}{\pi s}\right] .
\end{aligned}
$$

A factor $\frac{1}{2}$ appears for fermion dark matter which is composed of particle and anti-particle simultaneously, and the annihilation only occurs between particle and its anti-particle (similarly, the factor $\frac{1}{2}$ exists for complex scalar DM, while this factor is equal to 1 for real scalar, Majorana fermion DM). $s=\left(p_{1}+p_{2}\right)^{2}, M$ is the mass of the intermediate boson, and $\Gamma$ is the total width of the intermediate boson. $s_{1}, s_{2}$ are the darkon spin projections. $\tilde{\Gamma}_{f}$ is the rate of the virtual boson transiting into SM fermions (quarks or leptons), to obtain it, one only needs to replace the intermediate boson mass by $\sqrt{s}$ in the calculations. $N_{c}$ is the color factor. $c_{a}$ is the axial vector current parameter, here $c_{a}^{2}=1$. $\beta_{i}=\sqrt{1-4 m_{D}^{2} / s}, \beta_{f}=\sqrt{1-4 m_{f}^{2} / s}$ are the kinematic factors.

In the case of $m_{Z^{\prime}} \gg m_{Z^{0}}$, the annihilation of a darkon pair into SM particles is dominated by $Z^{0}$ with the mixing component, namely via darkon + darkon $\rightarrow Z^{0} \rightarrow S M$. Using formula (23), we can get the annihilation cross section. The DM relic density $\Omega_{D}$ is determined by the thermal dynamics of the big-bang cosmology. The approximate values of the relic density and freeze-out temperature are [37, 38]

$$
\begin{gathered}
\Omega_{D} h^{2} \simeq \frac{1.07 \times 10^{9} G e V^{-1} x_{f}}{\sqrt{g_{*}} m_{\mathrm{Pl}}\left\langle\sigma_{\mathrm{ann}} v_{\mathrm{rel}}\right\rangle}, \\
x_{f} \simeq \ln \frac{0.038 g m_{\mathrm{Pl}} m_{D}\left\langle\sigma_{\mathrm{ann}} v_{\mathrm{rel}}\right\rangle}{\sqrt{g_{*} x_{f}}} .
\end{gathered}
$$

Here $h$ is the Hubble constant in units of $100 \mathrm{~km} /(\mathrm{s} \cdot \mathrm{Mpc})$, and $m_{\mathrm{Pl}}=1.22 \times 10^{19} \mathrm{GeV}$ is the Planck mass. $x_{f}=m_{D} / T_{f}$ with $T_{f}$ being the freezing temperature, $g_{*}$ is the number of relativistic degrees of freedom with masses less than $T_{f} .\left\langle\sigma_{\text {ann }} v_{\text {rel }}\right\rangle$ is the thermal average of the annihilation cross section of DM pair transiting into SM particles and $v$ is the relative speed of the DM pair in their center of mass frame, and $g$ is the number of degrees of freedom of DM. In this work, the DM particle is assumed to be the darkon, which we describe above. The thermal average of the effective cross section is [39]

$$
\left\langle\sigma_{a n n} v_{r e l}\right\rangle=\frac{1}{8 m_{D}^{4} T K_{2}^{2}\left(\frac{m_{D}}{T}\right)} \int_{4 m_{D}^{2}}^{\infty} d s \sigma_{a n n} \sqrt{s}\left(s-4 m_{D}^{2}\right) K_{1}\left(\frac{\sqrt{s}}{T}\right)
$$




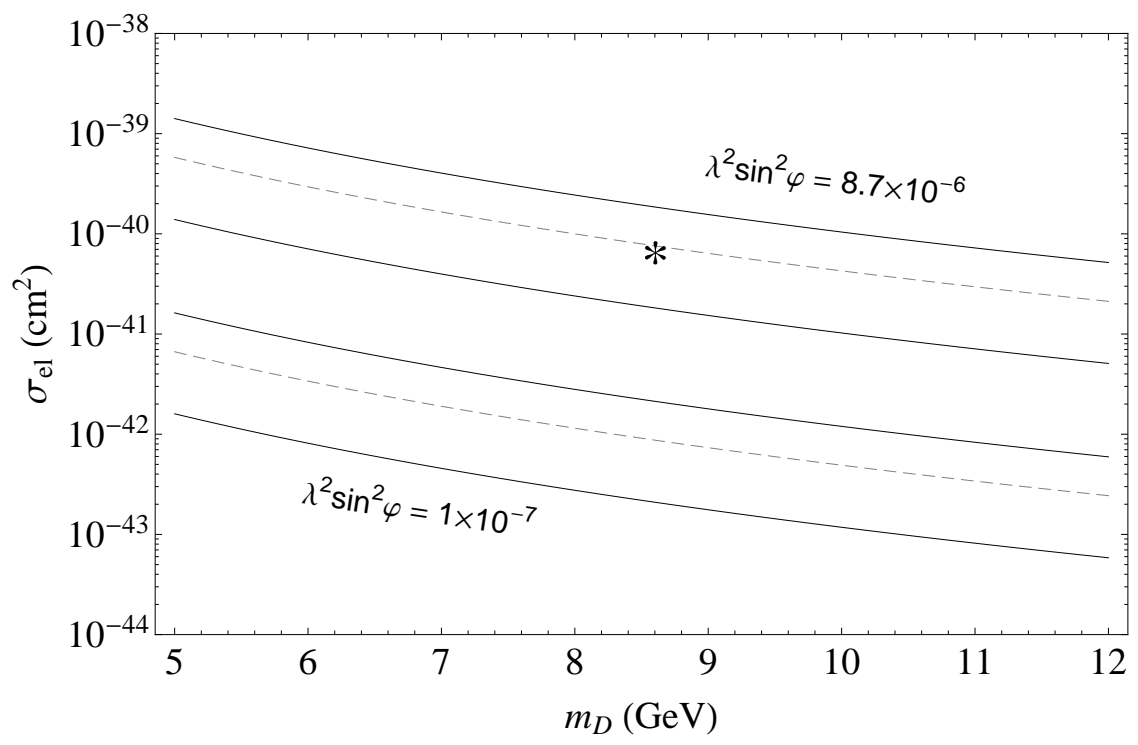

FIG. 2: Darkon-neutron SI elastic cross-section $\sigma_{\text {el }}$ as a function of the darkon's mass. $m_{D}$ varies in a range $5 \mathrm{GeV} \leq m_{D} \leq 12 \mathrm{GeV} .2 m_{D} / m_{Z^{\prime}}=\xi$, for $\xi$ equal to $0.7,1,1.25 . \lambda^{2} \sin ^{2} \varphi=8.7 \times 10^{-6}, 1 \times 10^{-7}$. The dashed curve is in the case $\xi=1$, the upper solid curve is $\xi=1.25$, and the the lower solid curve is $\xi=0.7$. The $*$ is the reserved CDMS II observed event.

where $K_{i}(x)$ is the modified Bessel functions of order $i$.

We calculate the cross section of low mass darkon pairs (the mass of darkon is supposed to be of order $10 \mathrm{GeV}$ ) annihilating into SM leptons and quarks (except top quark) via $Z^{0}$ exchanged. $x_{f}$ is obtained by solving the Eq. (25) iteratively. The effective degrees of freedom $g_{*}$ is varying with the freeze-out temperature $T_{f}$, and we take the data of Gondolo-Gelmini effective degrees of freedom in MicrOMEGAs 3.1 at $T_{Q C D}=150 \mathrm{MeV}$ [40]. For $m_{D} \sim 8.6 \mathrm{GeV}$, the DM density is $\Omega_{D} h^{2} \approx 0.593$. The current PDG value for cold DM density is $\Omega_{c d m} h^{2}=0.111(6)$ [36]. Thus in the case of $m_{Z^{\prime}} \gg m_{Z^{0}}$, the DM relic density is superabundant. Therefore this scenario is not consistent with both the LEP data and the observed DM relic density, so that should be abandoned.

Below we turn to another possibility that $m_{Z^{\prime}} \ll m_{Z^{0}}$.

\section{The case $m_{Z^{\prime}} \ll m_{Z^{0}}$}

Now, let us consider the case of $m_{Z^{\prime}} \ll m_{Z^{0}}$. If the pole mass of $Z^{\prime}$ is just slightly above $2 m_{D}$, the annihilation cross section of darkon pair can be enhanced. The darkon-nucleon elastic scattering occurs mainly via exchanging $Z^{\prime}$ in this case, and the cross section is similar to the case for $m_{Z^{\prime}} \gg m_{Z^{0}}$ and can be re-written as

$$
\sigma_{\mathrm{el}} \simeq G_{F} \frac{m_{z^{0}}^{2}}{m_{z^{\prime}}^{2}} \frac{\sqrt{2} \lambda^{2} \sin ^{2} \varphi m_{D}^{2} m_{N}^{2}\left(3 G_{A}^{z 2}+F_{1}^{z^{2}}\right)}{4 \pi\left(p_{D}+p_{N}\right)^{2} m_{z^{\prime}}^{2}} .
$$




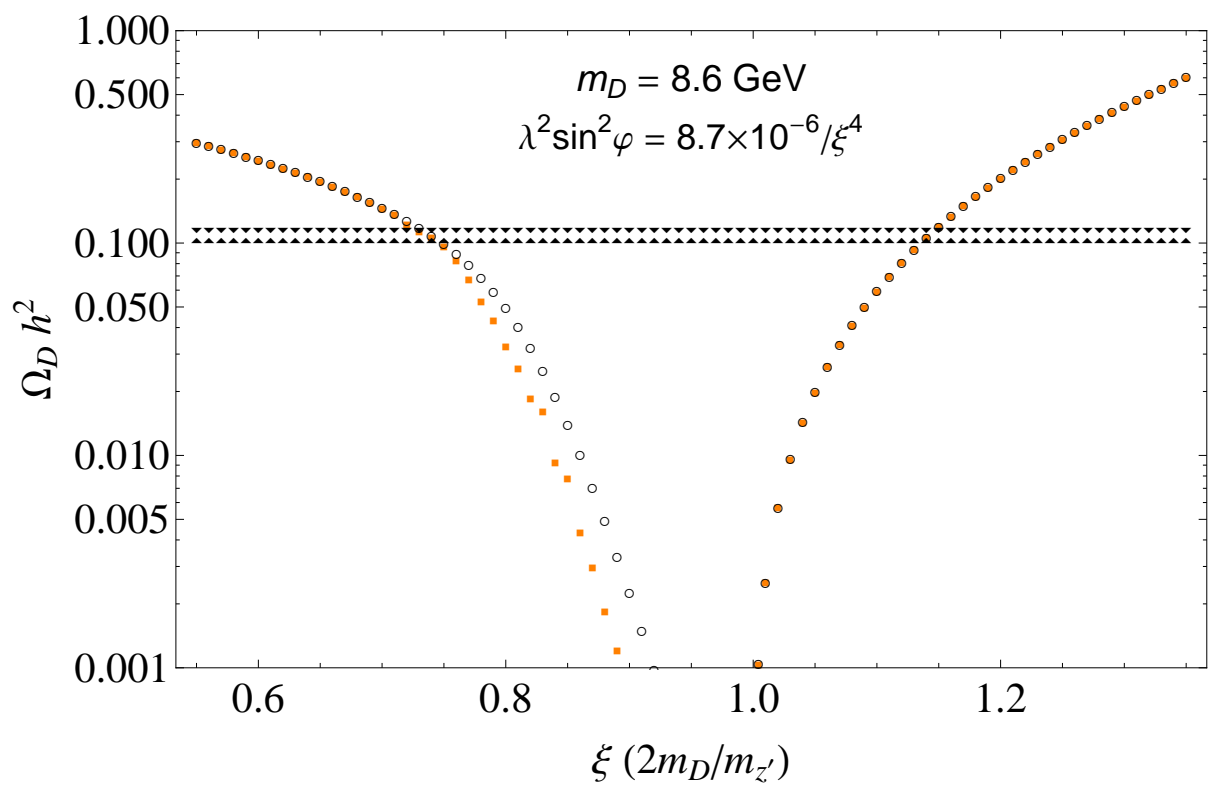

FIG. 3: Darkon relic density $\Omega_{D} h^{2}$ as a function of $\xi\left(2 m_{D} / m_{Z^{\prime}}\right)$ near the $Z^{\prime}$ pole when $m_{D}=8.6$ $\mathrm{GeV}$, for $\xi$ in a range from 0.55 to 1.35 and $\lambda^{2} \sin ^{2} \varphi=8.7 \times 10^{-6} / \xi^{4}$. The solid square curve is in the case $\lambda=0.5$, and the empty dotted curve is $\lambda=1.0$. The triangle and triangle-down curves are the cold dark matter relic density $0.111(6)$ today.

Taking the CDMS II results for darkon-neutron elastic scattering as our inputs, we get $\lambda^{2} \sin ^{2} \varphi \approx 6.88 \times 10^{-3} \times\left(m_{z^{\prime}}^{4} / m_{z^{0}}^{4}\right)$. As $\cos \varphi \sim 1$, the width of $Z^{\prime}$ decaying into a darkon pair is

$$
\Gamma_{D}^{\prime} \simeq \frac{\lambda^{2}\left(m_{z^{\prime}}^{2}-m_{D}^{2}\right)}{24 \pi m_{z^{\prime}}} \sqrt{1-\frac{4 m_{D}^{2}}{m_{z^{\prime}}^{2}}} .
$$

For the LEP constraint, using formula (21) and rewriting formula (22), we can obtain that when $\left(m_{z^{\prime}}^{4} / m_{z^{0}}^{4}\right)<0.167$, the width of $Z^{0}$ decaying into neutrinos plus darkons is within the experimental tolerance range. This can be satisfied when $Z^{\prime}$ is lighter than half of the $Z^{0}$ mass.

At the leading order, the annihilation of a darkon pair into SM particles is determined by $Z^{\prime}$ with the mixing component and the cross section is calculated by formula (23). When $m_{Z^{\prime}}<2 m_{D}$, the annihilation of a darkon pair into SM particles can also pass the constraints set by the aforementioned collider experiment and astronomical observation.

Define $2 m_{D} / m_{Z^{\prime}}=\xi$. By fitting the data, in the case the $Z^{\prime}$ mass is near $2 m_{D}$, we obtain $\lambda^{2} \sin ^{2} \varphi \simeq 8.7 \times 10^{-6}(\xi=1)$ in the darkon-neutron SI elastic cross-section. The dependence of the elastic scatting cross section on $m_{D}$ is shown in Fig. 2, where $m_{D}$ varies within a range of $5 \mathrm{GeV} \leq m_{D} \leq 12 \mathrm{GeV}$ and $\xi$ takes the values of $0.7,1,1.25 . \lambda^{2} \sin ^{2} \varphi=1 \times 10^{-7}$ is given as a comparison. For $m_{D} \sim 8.6 \mathrm{GeV}$, fitting the results of CDMS, we have $\lambda^{2} \sin ^{2} \varphi \simeq 8.7 \times 10^{-6} / \xi^{4}$.

The dependence of the darkon relic density $\Omega_{D} h^{2}$ on $\xi\left(2 m_{D} / m_{Z^{\prime}}\right)$ is depicted in Fig. [3] where $m_{D}$ is set to be $8.6 \mathrm{GeV}$ and $\xi$ varies from 0.55 to $1.35 . \lambda^{2} \sin ^{2} \varphi \simeq 8.7 \times 10^{-6} / \xi^{4}$. The solid square, empty dotted curves are for $\lambda=0.5,1.0$ respectively. When $\xi>1$, the curve for $\lambda=0.5$ 


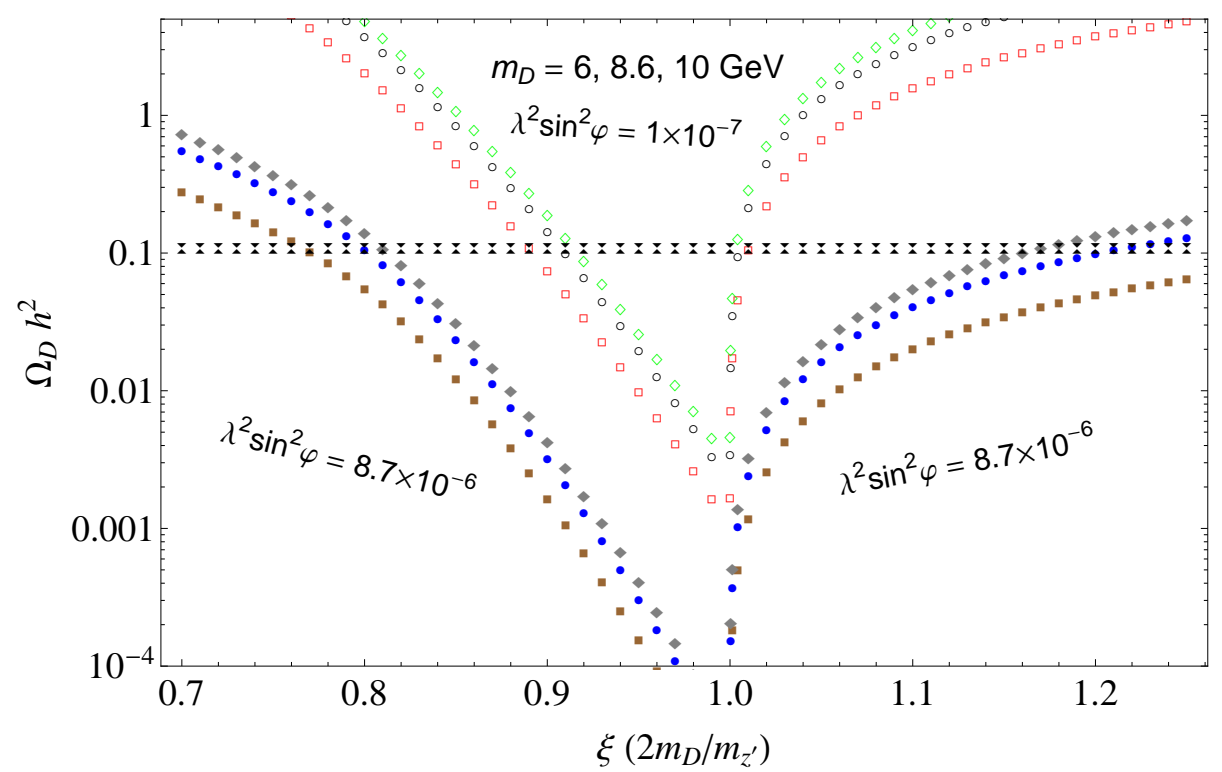

FIG. 4: Darkon relic density $\Omega_{D} h^{2}$ as a function of $\xi\left(2 m_{D} / m_{Z^{\prime}}\right)$ near the $Z^{\prime}$ pole when $m_{D}=6$, 8.6, $10 \mathrm{GeV}$, for $\xi$ 's values varying from 0.7 to 1.25 . $\lambda=1$ is taken here. The solid curves are for the case $\lambda^{2} \sin ^{2} \varphi=8.7 \times 10^{-6}$, and empty curves are for the case $\lambda^{2} \sin ^{2} \varphi=1 \times 10^{-7}$. The square curves, dotted curves and diamond curves (solid, empty) are corresponds to the case $m_{D}$ equal to $6,8.6$ and $10 \mathrm{GeV}$ respectively. The triangle and triangle-down curves are the cold dark matter relic density $0.111(6)$.

is close to the curve for $\lambda=1.0$. It can be seen that, there is a parameter space allowed by the present data.

As a comparison, the dependence of the darkon relic density $\Omega_{D} h^{2}$ on $m_{D}$ and $\lambda^{2} \sin ^{2} \varphi$ is shown in Fig. 4 where $m_{D}$ is set as $6,8.6,10 \mathrm{GeV}$ and $\lambda^{2} \sin ^{2} \varphi=8.7 \times 10^{-6}, 1 \times 10^{-7}$. We take $\lambda=1$ here and let $\xi$ vary from 0.7 to 1.25 . The solid curves, empty curves are for the case $\lambda^{2} \sin ^{2} \varphi=8.7 \times 10^{-6}, 1 \times 10^{-7}$ respectively. The square curves, dotted curves and diamond curves are corresponding to the case $m_{D}$ equal to $6,8.6$ and $10 \mathrm{GeV}$ respectively.

\section{B. Scalar and vector darkons}

Now let us consider the scalar-darkon case. The effective vertex is a vector coupling $-i \lambda(k+$ $\left.k^{\prime}\right)^{\mu}$, as shown in Fig. 5 (left). As aforementioned, the scattering of darkon-nucleon scattering induced by this interaction is a unsuppressed SI process [30]. In the limit $\frac{P^{\mu}}{m} \rightarrow(1, \epsilon)$, the darkon-nucleon elastic scattering cross section by exchanging $Z^{0}$ is written as

$$
\sigma_{\mathrm{el}} \simeq \frac{\sqrt{2} G_{F} \lambda^{2} \sin ^{2} \varphi m_{D}^{2} m_{N}^{2} F_{1}^{z 2}}{\pi\left(p_{D}+p_{N}\right)^{2} m_{Z^{0}}^{2}} .
$$

$F_{1}^{z}=0.5-2 \sin ^{2} \theta_{w}(-0.5)$ for proton (neutron). Thus, the darkon-neutron scattering is dominant. Similar results can be obtained as for the fermionic darkon case. 

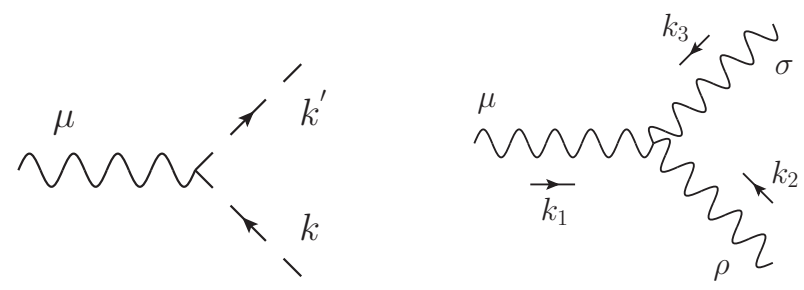

FIG. 5: Vertexes of scalar (left) and vector (right) darkons.

Instead, for the case that $Z^{\prime}$ exchanging is dominant, one can modify the formula (29) by simply multiplying a factor $m_{z^{0}}^{4} / m_{z^{\prime}}^{4}$.

For the vector darkon, the vertex is $-i \lambda\left[g^{\mu \rho}\left(k_{2}-k_{1}\right)^{\sigma}+g^{\rho \sigma}\left(k_{3}-k_{2}\right)^{\mu}+g^{\sigma \mu}\left(k_{1}-k_{3}\right)^{\rho}\right]$, corresponding to the effective interaction $B_{\mu}^{\dagger} \partial^{\nu} B^{\mu} \bar{q} \gamma_{\nu} q$, as shown in Fig. 5 (right) which contributes an unsuppressed SI cross section. In the limit $\frac{P^{\mu}}{m} \rightarrow(1, \epsilon)$, the darkon-nucleon elastic scattering cross section with $Z^{0}$ exchange-dominance can be written as

$$
\sigma_{\mathrm{el}} \simeq \frac{\sqrt{2} G_{F} \lambda^{2} \sin ^{2} \varphi m_{D}^{2} m_{N}^{2} F_{1}^{z^{2}}}{\pi\left(p_{D}+p_{N}\right)^{2} m_{Z^{0}}^{2}} .
$$

In the case that $Z^{\prime}$ exchange is dominant, the elastic cross section can be obtained by multiplying formula (30) by a factor $m_{z^{0}}^{4} / m_{z^{\prime}}^{4}$. The obtained result is the same as that for scalardarkon-nucleon elastic scattering.

It is noted, for fermionic, scalar and vector darkon-nucleon elastic scattering via exchanging $Z$-boson, there exists SI darkon-neutron scattering which are not suppressed by either $q^{2}$ or $v^{2}$. In this case, the proton contributions are suppressed, so that the main contributions to the SI scattering comes from the interaction between the darkon and neutron. Therefore, the xenon target which has more neutrons than protons is more sensitive compared with the silicon and germanium targets. As explained above, if we accept the claim of XENON10 [41] and XENON100 [2] that for low energy WIMPs, null results have been obtained, the CDMS results should be dubious. But suggested by Hooper [3], a re-analysis may imply that the peculiar events observed at the XENON100 might be explained as dark matter candidates to be reconciled with the CDMS data. With the further progress of the XENON experiments, more information will be obtained for the low mass WIMPs with masses of order $10 \mathrm{GeV}$.

\section{CONCLUSIONS AND DISCUSSION}

Taking the recent new results of the CDMS II experiments searching for WIMPs with masses of order $10 \mathrm{GeV}$ as inputs and considering some constraints from LHC, LEP and astronomical observation etc. altogether, we discuss a simple WIMP candidate: the darkon which can be scalar, fermion or vector. We have found that in the simplest scenario of the standard model plus a SM singlet DM (the darkon), one cannot simultaneously satisfy the CDMS II's observation and the LHC data, and this result is consistent with the former result implied in [7]. Thus, one 
must extend the SM to include new physics beyond the standard model. Here we consider the extended gauge group $S U_{L}(2) \otimes U_{Y}(1) \otimes U(1)^{\prime}$ which later breaks into $U_{e m}(1)$ to result in two heavy neutral gauge bosons $Z^{0}$ and $Z^{\prime}$.

The darkon $+\mathrm{SM}+U(1)^{\prime}$ scenario must undergo stringent tests from the cosmology observation and the LHC data. Namely, all the CDMS II results, dark matter density in our universe and the data of $Z^{0}$ decaying into invisible products which were obtained by LEP experiments must not conflict.

Our numerical results indicate that in this scenario, only if $Z^{\prime}$ is lighter than $Z^{0}$, all the constraints can be satisfied. Under this assumption, the model darkon $+\mathrm{SM}+U(1)^{\prime}$ withstand all the constraints set by the presently available data. Moreover, it is noted that as long as $m_{Z^{\prime}} \sim 2 m_{D}$, the model can accommodate even smaller scattering cross section and lighter darkons.

Indeed we should further test the validity of this mechanism. If in the future, we can precisely measure the branching ratios of heavy quarkonia, such as botomonia decaying into invisible products, or the invisible decays of the SM Z boson and Higgs sector physics, we would be able to determine which one e.g., of the two-Higgs-doublets mechanism or the extra $U(1)^{\prime}$ gauge group, is more reasonable. We lay hope on the future more precise detection on the dark matter, no matter direct or indirect.

In the world today there are many laboratories directly searching for dark matter besides the XENON and CDMS collaborations, for example, the China Jin-Ping underground laboratory [42] just joined the club and the China Dark-Matter experiment (CDEX) is using $1 \mathrm{~kg}$ Ge detector and will develop $10 \mathrm{~kg}$ and 1 ton detector for the project. We are expecting that the world-wide cooperation can eventually reveal the Epoch mystery.

\section{Acknowledgments}

We thank Prof. X.G. He for helpful discussions. This work was partially supported by National Natural Science Foundation of China under the contract No.11075079, 11135009.

[1] R. Agnese et al. [CDMS Collaboration], arXiv:1304.4279 [hep-ex]].

[2] E. Aprile et al. [XENON100 Collaboration], Phys. Rev. Lett. 109 (2012) 181301 [arXiv:1207.5988 [astro-ph.CO]].

[3] D. Hooper, JCAP 1309, 035 (2013) [arXiv:1306.1790 [hep-ph]].

[4] V. Silveira and A. Zee, Phys. Lett. B 161 (1985) 136.

[5] J. McDonald, Phys. Rev. D 50 (1994) 3637 hep-ph/0702143 [HEP-PH]].

[6] C. P. Burgess, M. Pospelov and T. ter Veldhuis, Nucl. Phys. B 619 (2001) 709 hep-ph/0011335.

[7] X. -G. He, T. Li, X. -Q. Li and H. -C. Tsai, Mod. Phys. Lett. A 22 (2007) 2121 hep-ph/0701156.

[8] X. -G. He, T. Li, X. -Q. Li, J. Tandean and H. -C. Tsai, Phys. Rev. D 79 (2009) 023521 arXiv:0811.0658 [hep-ph]].

[9] Y. Mambrini, Phys. Rev. D 84 (2011) 115017 [arXiv:1108.0671 [hep-ph]].

[10] G. Aad et al. [ATLAS Collaboration], Phys. Lett. B 716 (2012) 1 [arXiv:1207.7214 [hep-ex]]. 
[11] S. Chatrchyan et al. [CMS Collaboration], Phys. Lett. B 716 (2012) 30 arXiv:1207.7235 [hep-ex]].

[12] X. -G. He and J. Tandean, Phys. Rev. D 88 (2013) 013020 arXiv:1304.6058 [hep-ph]].

[13] O. Lebedev, H. M. Lee and Y. Mambrini, Phys. Lett. B 707 (2012) 570 [arXiv:1111.4482 [hep-ph]].

[14] A. Djouadi, O. Lebedev, Y. Mambrini and J. Quevillon, Phys. Lett. B 709 (2012) 65 arXiv:1112.3299 [hep-ph]].

[15] L. Resnick, M. K. Sundaresan and P. J. S. Watson, Phys. Rev. D 8 (1973) 172.

[16] J. R. Ellis, M. K. Gaillard and D. V. Nanopoulos, Nucl. Phys. B 106 (1976) 292.

[17] K. -Y. Choi and O. Seto, Phys. Rev. D 88 (2013) 035005 arXiv:1305.4322 [hep-ph]].

[18] D. E. Brahm and L. J. Hall, Phys. Rev. D 41, 1067 (1990).

[19] B. de Carlos and J. R. Espinosa, Phys. Lett. B 407, 12 (1997) hep-ph/9705315.

[20] J. Erler, P. Langacker and T. -j. Li, Phys. Rev. D 66, 015002 (2002) [hep-ph/0205001].

[21] B. Kors and P. Nath, Phys. Lett. B 586, 366 (2004) hep-ph/0402047].

[22] V. Barger, C. Kao, P. Langacker and H. -S. Lee, Phys. Lett. B 600, 104 (2004) hep-ph/0408120.

[23] B. Kors and P. Nath, JHEP 0507, 069 (2005) hep-ph/0503208].

[24] P. Langacker, Rev. Mod. Phys. 81 (2009) 1199 [arXiv:0801.1345 [hep-ph]].

[25] J. Kumar and J. D. Wells, Phys. Rev. D 74, 115017 (2006) hep-ph/0606183.

[26] W. -F. Chang, J. N. Ng and J. M. S. Wu, Phys. Rev. D 74, 095005 (2006) [Erratum-ibid. D 79, 039902 (2009)] [hep-ph/0608068].

[27] D. Feldman, Z. Liu and P. Nath, Phys. Rev. D 75, 115001 (2007) hep-ph/0702123 [HEP-PH]].

[28] Y. Mambrini, JCAP 1009 (2010) 022 [arXiv:1006.3318 [hep-ph]].

[29] M. T. Frandsen, F. Kahlhoefer, S. Sarkar and K. Schmidt-Hoberg, JHEP 1109 (2011) 128 arXiv:1107.2118 [hep-ph]].

[30] M. Freytsis and Z. Ligeti, Phys. Rev. D 83 (2011) 115009 arXiv:1012.5317 [hep-ph]].

[31] M. C. Smith, G. R. Ruchti, A. Helmi, R. F. G. Wyse, J. P. Fulbright, K. C. Freeman, J. F. Navarro and G. M. Seabroke et al., Mon. Not. Roy. Astron. Soc. 379 (2007) 755 [astro-ph/0611671].

[32] G. Garvey, E. Kolbe, K. Langanke and S. Krewald, Phys. Rev. C 48 (1993) 1919.

[33] G. T. Garvey, Prog. Part. Nucl. Phys. 34 (1995) 245.

[34] W. M. Alberico, S. M. Bilenky and C. Maieron, Phys. Rept. 358 (2002) 227 hep-ph/0102269.

[35] H. Forkel, Phys. Rev. C 56 (1997) 510 hep-ph/9607452.

[36] J. Beringer et al. [Particle Data Group Collaboration], Phys. Rev. D 86 (2012) 010001.

[37] E. W. Kolb and M. S. Turner, The Early Universe (Westview Press, Boulder, 1990).

[38] K. Griest and D. Seckel, Phys. Rev. D 43 (1991) 3191.

[39] P. Gondolo and G. Gelmini, Nucl. Phys. B 360 (1991) 145.

[40] G. Belanger, F. Boudjema, A. Pukhov and A. Semenov, arXiv:1305.0237 [hep-ph]. See also, http://lapth.cnrs.fr/micromegas/

[41] J. Angle et al. [XENON10 Collaboration], Phys. Rev. Lett. 107 (2011) 051301 arXiv:1104.3088 [astro-ph.CO]].

[42] K. -J. Kang et al. [CDEX Collaboration], Front. Phys. China. 8 (2013) 412 arXiv:1303.0601 [physics.ins-det]]. 